\title{
Research Paper: The Effect of 8 Weeks Virtual Reality Training on Static and Dynamic Balance and Performance in Male Athletes With Functional Ankle Instability
}

\author{
Farhad Ranjbarzadeh Yamchi ${ }^{*}$ (D), Amir Letafatkar² ${ }^{2}$, Saeed Esmaeilpour ${ }^{2}$ (D) \\ 1. Department of Sport Pathology and Corrective Exercises, Faculty of Physical Education and Sport Sciences, University of Tehran, Tehran, Iran. \\ 2. Department of Sport Pathology and Corrective Exercises, Faculty of Physical Education and Sport Sciences, University of Kharazmi, Tehran, Iran
}

\begin{tabular}{|c|c|}
\hline $\begin{array}{l}\text { Use your device to scan } \\
\text { and read the article online }\end{array}$ & \\
\hline 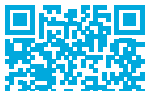 & $\begin{array}{l}\text { Dynamic Balance and Performance in Male Athletes With Functional Ankle Instability. Physical Treatments. 2021; 11(1):45-54. } \\
\text { http://dx.doi.org/10.32598/ptj.11.1.453.1 }\end{array}$ \\
\hline 口ifting & dol': http://dx.doi.org/10.32598/ptj.11.1.453.1 \\
\hline
\end{tabular}

\section{(i) (3)}

Article info:

Received: 29 Mar 2020

Accepted: 14 Sep 2020

Available Online: 01 Jan 2021

Keywords:

Virtual reality, Virtual reality exercise, Balance, Performance

\section{A B S T RA C T}

Purpose: The purpose of this study is to investigate the effect of 8 weeks virtual reality training on static and dynamic balance and performance of in male athletes with functional ankle instability

Methods: or this research, 30 volleyball, basketball, football and handball players ranged from 18 to 25 years were selected purposefully from Tehran province league male athletes based on inclusion and Exclusion criteria. Then Subjects were randomly divided into two groups included 15 subjects of virtual reality exercises and control group. After a 5 minute warm-up program, the static and dynamic balance and performance were measured by stork test, y balance test and side jump and triple jump test respectively. Then, training group completed an 8-week (24 sessions) exercise program. Finally, after completing the training protocol, a similar post-test with pre-test was done. To test and analyze datum, also analysis, paired t-test and ANCOVA statistical method was used.

Results: Paired t-test and analysis of covariance showed that there was a significant difference in all variables in the post-test compared to the pre-test and also between the two groups $(\mathrm{P}<0.50)$. The balance of subjects and their performance in the experimental group was improved

Conclusion: The 8 weeks of virtual reality training significantly improve balance and performance of male with functional ankle instability; therefore coaches and athletes can use this exercises to improve balance and performance in athletes with functional ankle instability.

\section{"Corresponding Author:}

Farhad Ranjbarzadeh Yamchi, PhD. Student

Address: Department of Sport Pathology and Corrective Exercises, Faculty of Physical Education and Sport Sciences, University of Tehran, Tehran, Iran. Phone: +98 (992) 1460910

E-mail: farhad.ranjbar8@gmail.com 


\section{Highlights}

- Virtual reality-based exercises have the ability to improve static and dynamic balance and performance of athletes with functional ankle sprains.

\section{Plain Language Summary}

People who suffer from recurrent ankle sprains are said to have ankle instability. Finding a solution to this problem seems necessary. Thirty semi-amateur athletes present in the leagues of Tehran Province from the volleyball, basketball, football, and futsal leagues were randomly selected according to the selection and exclusion criteria and were divided into a control group and an experimental group (exercises based on virtual reality). The experimental group performed virtual reality-based exercises for 8 weeks and 3 sessions per week, under the supervision of the researcher. According to the results of this study, virtual reality-based exercises have a significant effect on static and dynamic balance and performance of athletes, and these exercises can be used to improve static and dynamic balance, as well as the performance of basketball, volleyball, football and futsal players. The use of virtual reality and games is one of the new ways to use technology in order to rehabilitate people with balance disorders and postural control, which in addition to providing rehabilitation facilities and required movement and functional activities, these facilities in the environment provides a home and eliminates the need for them to go to the relevant clinics. The attractiveness and novelty of these exercises make athletes and coaches more willing to use these exercises than traditional exercises.

\section{Introduction}

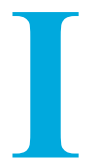

$\mathrm{n}$ proportion to the increasing tendency towards exercise, musculoskeletal injuries that are an inevitable part of the exercise have also increased [1]. Also, the complete elimination of sports injuries is impossible. However, the prevalence and severity of injuries can be significantly reduced by appropriate strategies.

Studies indicate that most of the sports injuries are associated with lower limbs [2]. The ankle is the most common injury site of the body, accounting for $25 \%$ of all sports injuries [3]. Patients who suffer from recurrent ankle sprains are said to have ankle instability. Ankle instability exists both mechanically and functionally. Freeman first defined functional instability as the foot's tendency to twist frequently or muscle fatigue [4]. Functional instability is a different phenomenon from mechanical instability. However, both can exist simultaneously. Mechanical instability refers to joint movement beyond the normal physiological range, while in functional instability, the joint movement goes beyond the individual's voluntary control in the physiological range [5].

Among ankle injuries, the external ligament springs account for $85 \%$ of all injuries. Also, external ankle sprains have obvious disturbing consequences, including functional instability ( $70 \%$ of cases), the impairment of postural oscillation and balance, the weakness of surrounding muscles, the dysfunction of neuromuscular muscles, proprioception impairment, the decreased range of motion, wrist joint flexion, and increased athlete reaction time [1].

In this regard, Delahunt et al. mentioned that patients with functional ankle instability showed changes in muscle activation and movement patterns in areas farther from the site of the original injury. Also, these people show altered walking kinematics, which is probably caused by compensatory changes in the feedforward control of the motor program [6].

Various studies have examined the use of conservative therapies, including balance exercises, neuromuscular training with balance board, strength training, plyometric training, and core stability training [7-12]. These exercises primarily aimed to improve neuromuscular function and the kinetics and kinematics of the ankle joint. A literature review suggested that these exercises could not reduce the prevalence of ankle sprain injury as it should. One of these exercises is virtual reality-based training that is a new technology emphasizing the graphical environment. This training not only creates the feeling of being in a physical environment but also simulates the interaction of the person with that environment [13]. This technology can increase the quality of life and improve individuals' health and functional abilities because it enhances individuals ability to perform various daily activities [14]. According to studies, virtual reality and games are methods that use new technology to rehabilitate people with disabilities, children, and the elderly 
with impaired balance and postural control. Besides, these exercises eliminate commuting to relevant clinics and provide rehabilitation facilities and movement and functional activities required by these people [15-19]. One of the games designed in this regard is the Nintendo Wii Fit. The Nintendo device puts a pleasant image with a resolution of $1080 \times 1920$ in front of the user. The device is equipped with five USB ports, a unique port for Kinect, HDMI and AV outputs, Toslink output, and WiFi wireless network. The Wii Fit game presents many balance challenges to the user. In this regard, studies investigated the effect of virtual reality-based training on upper and lower limb rehabilitation, in the elderly group with mobility problems and mental disabilities. All the studies have reported the usefulness of these exercises in the rehabilitation phase $[15,20]$. Despite all the benefits of using this training method, studies are limited to a handful of athletes, especially in injury prevention and returning to exercise after an injury, which is the primary goal of sports pathology [21]. It raises a question in the mind: compared with the other standard methods of training, whether the use of these exercises is effective in preventing or safely returning athletes with functional ankle instability to exercise?

For example, Kim et al. compared the effect of virtual reality-based training and traditional rehabilitation training on athletes with functional ankle instability. The virtual reality exercise group showed more power than the traditional rehabilitation exercise group, only in the plantarflex motion. Also, in other movements, the muscle strength of the virtual reality exercise group was higher than that of the rehabilitation exercises group. However, both training groups revealed a significant improvement, compared with the control group [22]. Babadi et al. also examined the effect of a virtual exercise program on motor coordination in the elderly. The authors concluded that virtual exercises improved motor coordination in the elderly, and virtual reality-based training could be used as a new and attractive training method to improve the elderly's coordination, in the daily programs of nursing homes [23].

On the other hand, previous studies show that it is challenging to convince coaches and players to solely carry out preventive programs to prevent injuries unless performing these exercises directly impact player performance [24]. Therefore, the best way to prevent injuries is to design a comprehensive program that emphasizes improving the performance of athletes and preventing injuries. Thus, it seems necessary to investigate the effect of this training method, which is performed to prevent or rehabilitate ankle injuries, and especially, the functional instability injuries of the ankle and examine the effect of using this training method on variables, such as the balance and performance of athletes. Therefore, this study aimed to investigate the effect of eight weeks of virtual reality-based training on the static and dynamic balance and performance of male athletes with functional ankle instability.

\section{Materials and Methods}

The present research was an experimental study with a pre-test-post-test design that included an experimental group (virtual reality-based training) and a control group. The statistical population consisted of semi-amateur athletes in the leagues of volleyball, basketball, football, and futsal fields, in Tehran province. Based on the inclusion and exclusion criteria, 30 subjects were randomly selected from these athletes. They were randomly assigned into a control group and an experimental group (virtual reality-based training).

Inclusion criteria included the age range of 18 to 25 years; having at least two years of sports experience in volleyball, basketball, football, or handball; having functional ankle instability; a history of at least one external ankle sprain injury (which requires some time to protect against weight intolerance with inactivity), in the last two years; and being able to bear the weight fully during the study $[25,26]$. Exclusion criteria included pain in any part of the body during the test so that the subject is unable to cooperate [27], more than two sessions of absence in the training sessions [28], the history of nervous or atrial system disorders, a history of ligament or meniscus injury, and head trauma.

The study samples were randomly assigned into a virtual reality-based training group $(n=15)$ and a control group ( $\mathrm{n}=15)$, after identifying based on the inclusion and exclusion criteria. Also, the samples received the necessary explanations about the research and signed the consent form of voluntary and informed participation in the research. Then, based on a specific schedule, the participants were asked to attend the gymnasium of Kharazmi University dormitory to make pre-test measurements. After the subjects' presence, the initial information form was first completed, then, anthropometric measurements were performed on each person. The Stork balance test and the $\mathrm{Y}$ balance test were used to measure static and dynamic balances, respectively.

The Stork balance test was performed while the athlete stood on his injured leg and placed the sole of his uninjured foot next to the inside of his superior knee with the fingers facing down (Figure 1). For this test, the hands should be on the waist and the pelvic crown, then, the 


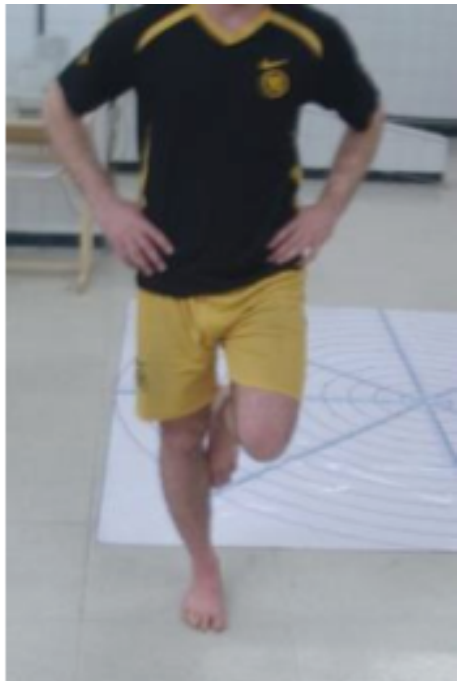

Figure 1. The Stork test

PHYSICAL TREA $\mid$ MENTS

subject is asked to close his eyes and lift the heel of the superior foot off the ground and stand on his superior plantar fascia, with the examiner's command. The duration of the test is recorded as the heel is lifted. If the subjects' balance is upset for any reason or they make a mistake, time is stopped and recorded. Each subject performed this test three times; the average of the three performances was considered as the final record for each subject. The validity and reliability of the test are 0.96 and 0.87 , respectively, which are acceptable [29].

For the Y balance test, three meters of tape measure were glued to the ground in three directions. So that, the zero meter mark was located in the center of the test site; one of the tape measures was directed anteriorly, and the other two tape measures had the angle of $135^{\circ}$ with the anterior tape measures. The subjects stood on their injured leg in the center of the test site to perform dynamic balance. Then, they tried to maintain their balance while trying to stretch the other foot as far as possible in three directions (anterior, posterior-interior, and posterior-exterior). When reaching maximum distance, the subject should slowly touch the tape measure with the reaching foot and return to the initial state (Figure 2). Then, the amount of reaching distance (normalized by dividing by the length of the foot and multiplying by 100) was recorded as the measure of performance [18]. As with the static balance test, each subject performed the test three times; the average of the three performances was considered as the final record. Pollock reported a very high reliability (0.8-0.95) for this test [30].

The performance of the subjects was also evaluated with the lateral jump and triple hop tests. The lateral jump test was used to measure the power, speed, balance,

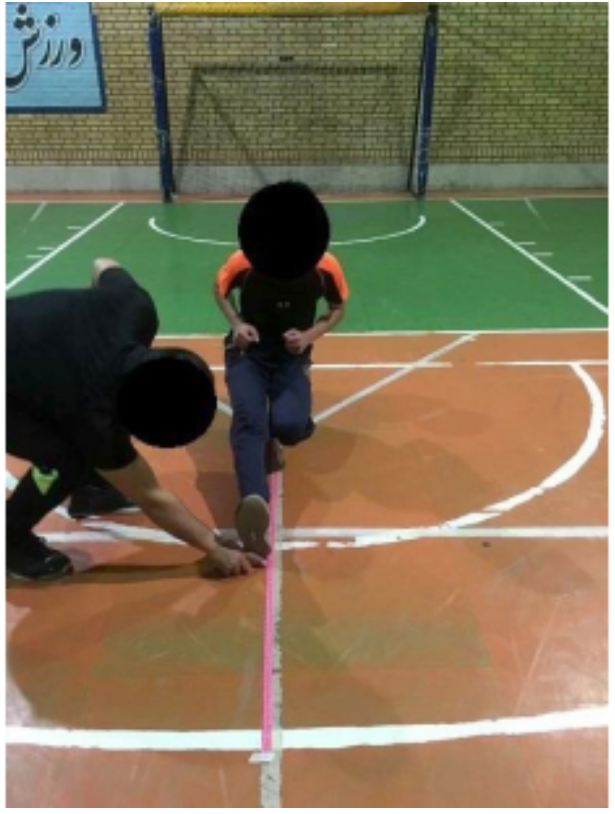

Figure 2. The $\mathrm{Y}$ balance test

PHYSICAL TREA $M$ MENTS

and rotational stability of the lower limb, emphasizing one-legged control [31]. Ortiz et al. reported the intertest reliability of 0.97 for the lateral jump test, in healthy athletes [31]. At this test, the subjects first stood next to the starting line, with the foot by which they intended to perform the test (injured leg). Then, they slightly bent their other leg from the hip and knee, so that, he would not hit the ground. Next, the subjects went back and forth 10 times at maximum speed. Besides, a distance of $30 \mathrm{~cm}$ was marked with two parallel pieces of paper glued on the ground in the form of hopping. Also, the elapsed time was recorded with an accuracy of 0.01 seconds as the performance score. Moreover, the subjects were asked to keep their hands on the iliac crest during the test to avoid oscillating hand movements (Figure 3).

The subjects performed this test with sneakers and made one to three attempts [32]. The subjects were asked to perform two performances with a 30 -second break to record the scores. The best record of the subject was used to analyze the data [32]. If the subject landed on the two parallel pieces of paper glued or lost his balance during the test, it would be considered an error, and the test was repeated [32].

The triple hop test is used to assess lower limb strength and power. Hamilton et al. reported the test-retest reliability of 0.98 for this test [33]. Hamilton has introduced the triple hop test as a highly validated test for predicting lower limb strength and power in healthy college athletes. Thus, it is considered a suitable test for preseason evaluations. At this test, the subjects stood with 


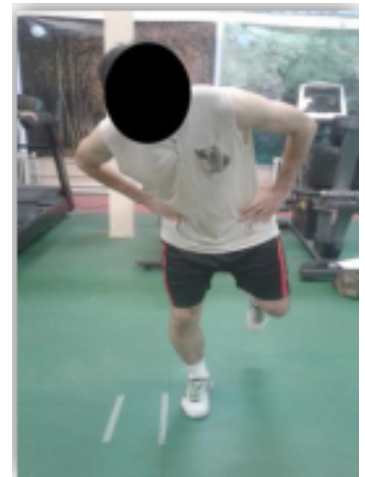

Figure 3. The lateral jump test

PHYSICAL TREATMENTS

the injured foot behind the starting line, so that their toes were behind the starting line (Figure 4). The subjects performed three maximum consecutive jumps with one leg in a straight line, also, their scores were calculated in centimeters, measuring from the starting line to the point where the heel hit the ground in the third jump. The subjects were asked to keep their hands on the iliac crest during the test and not use the arms' oscillating movements to jump further [33]. To perform the test and record the scores, the subject performed two performances with a 30 -second break. The best record of the subject was used to analyze the data [33].

The subjects were allowed to perform one to three attempts in all tests. After completing the pre-test measurements, the experimental group performed virtual realitybased training under the supervision of the researcher, for eight weeks and three sessions per week (Table 1).

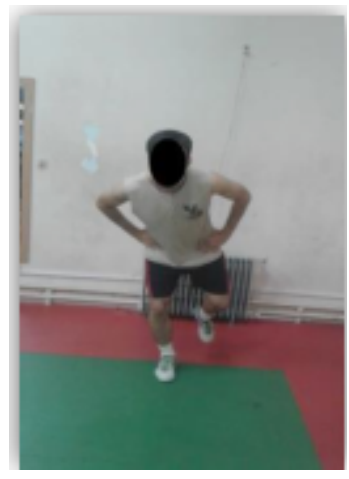

Figure 4. The triple hop test

PHYSICAL TREA $\ M E N T S$

Besides, the control group was asked not to do any specific sports activity during the study period and engage in their normal daily activities. Before starting the training course, a training session was held to familiarize the experimental group with virtual reality training using the Plus Nintendo Wii Fit (Nintendo Inc, Kyoto, Japan). The samples of the experimental group first warmed up their body for 10 minutes. Then, under the researcher's supervision, they performed 30 minutes of the selected strength and balance exercises, three sessions per week, for eight weeks. The warm-up included five minutes of riding on a stationary bike at a constant and predetermined speed (60 rpm with a resistance of $45 \mathrm{~W}$ ) and five minutes of the warm-up and stretches of the lower limb muscles.

A simple power plate is used to control the movement of the character in the game by transferring his/her weight on it, so that, the character in the game moves to

Table 1. Exercise protocol

\begin{tabular}{|c|c|c|}
\hline Weeks & Game/Movement & Set $\times$ Repetition \\
\hline \multirow{5}{*}{ Weeks 1 to 4} & Balance bubble & $3 \times 30$ \\
\hline & Sloping table & $3 \times 30$ \\
\hline & Dribble with the head & $3 \times 30$ \\
\hline & Hula-hoop & $3 \times 30$ \\
\hline & Gliding penguin & $3 \times 30$ \\
\hline \multirow{7}{*}{ Weeks 4 to 8} & Moving on a rope (rope walking) & $3 \times 30$ \\
\hline & Slalom skiing & $3 \times 30$ \\
\hline & Football match & $3 \times 30$ \\
\hline & & \\
\hline & Standing leg adduction (single-leg) & $3 \times 30$ \\
\hline & Rotating the thigh in and out (single-leg) & $3 \times 30$ \\
\hline & Squat & $3 \times 30$ \\
\hline
\end{tabular}


the right by transferring weight to the right. This video game includes games, such as hula-hooping, football, slack rope walking, skiing, and many yoga movements [22] (Figure 1). Such games and movements challenge the balance of the individual in various ways. One must carefully observe the movements of the character (the effective use of the visual system), interpret the observed images, and decide on the appropriate type of movement in response to these images to succeed in establishing and maintaining balance. The individual's symmetric equilibrium is tested, challenging the person to maintain their center of gravity and the center of balance at one point and using visual feedback to guide and control the character in the game [21].

The Shapiro-Wilk statistical test was used to check the normality of the data. Then, the descriptive statistics of mean and standard deviation were used to describe the information. Also, the paired t-test was used to compare the intragroup results, and the Analysis of Covariance (ANCOVA) was used to compare the intergroup results. All statistical analyses were performed at the significance level of 0.05, using SPSS V. 20.

\section{Results}

Table 2 reports the anthropometric characteristics related to the age, height, weight, and body mass index of the study subjects by the respective groups. The ShapiroWilk test indicated that the data were normally distributed. Therefore, parametric statistical methods were used to analyze the obtained data. A paired t-test was used to compare the intragroup results, and ANCOVA was used to compare the intergroup results in the post-test. The significance level of less than 0.05 was considered in all tests (Table 3). After six weeks of training, the paired t-test showed a significant difference between pre-test and post-test in static and dynamic balance and the performance of athletes in the experimental group. Moreover, the post-test performance was increased in the two groups, compared with the pre-test $(\mathrm{P} \geq 0.05)$. This result indicates an improvement in static and dynamic balance and performance in the experimental group, but no significant difference was observed in the control group, considering the mentioned variables $(\mathrm{P}<0.05)$. Besides, the ANCOVA was used to compare the difference between static and dynamic balance scores and the performance in the two groups; Table 3 reports the test results. The ANCOVA results showed a significant difference between the two research groups in all variables in the post-test, after controlling the effect of the pre-test (covariate) $(\mathrm{P}<0.05)$.

\section{Discussion}

This study aimed to compare the effect of eight weeks of virtual reality-based training on the static and dynamic balance and performance of male athletes with functional ankle instability. The present study results showed that the static and dynamic balance and performance of athletes significantly improved after eight weeks $(\mathrm{P}<0.05)$. The study of the effect size of virtual reality-based training by the eta-squared method indicated the great effect of these exercises on static and dynamic balance and performance. We tried to use the studies that had the closest results to the present study because virtual reality-based training is a new training method and research resources are not very rich. The results of the present study were in line with those of the Feng et al. [34], Kim et al. [22], and Babadi et al. [23], and no study contradicts the present study.

In a study on functional ankle sprains, Feng concluded that virtual reality-based training could be used to adjunct other exercise techniques by athletes [34]. Also, Kim et al. compared the effects of virtual reality-based training and traditional rehabilitation training on athletes with functional ankle instability. These authors concluded that only in the plantarflexion motion the virtual reality training group had more power than the traditional rehabilitation exercise group. In other muscle move-

Table 2. Descriptive statistics of the anthropometric characteristics of the study sample $(n=15)$

\begin{tabular}{|c|c|c|c|}
\hline \multirow{2}{*}{ Variables } & \multicolumn{2}{|c|}{ Mean $\pm S D$} & \multirow{2}{*}{$\mathbf{P}$} \\
\hline & Experimental Group & Control Group & \\
\hline Mean age (y) & $23.2 \pm 21.32$ & $24.1 \pm 74.75$ & 0.64 \\
\hline Mean height $(\mathrm{cm})$ & $175.1 \pm 10.15$ & $176.3 \pm 64.25$ & 0.92 \\
\hline Mean weight $(\mathrm{kg})$ & $70.4 \pm 61.02$ & $68.1 \pm 18.42$ & 0.13 \\
\hline BMI $\left(\mathrm{kg} / \mathrm{m}^{2}\right)$ & $23.07 \pm 1.19$ & $22.06 \pm 2.34$ & 0.11 \\
\hline
\end{tabular}


Table 3. Results of paired t-test and ANCOVA to examine intragroup and intergroup differences

\begin{tabular}{|c|c|c|c|c|c|c|}
\hline Variables & Control Group & $T(P)$ & Virtual Reality & $T(P)$ & $F(P)$ & Partial Eta-squared \\
\hline Static balance, $s$ & & $-0.65(0.52)$ & & $3.99(0.002) *$ & $23.04(0.001)^{*}$ & 0.58 \\
\hline Pre-test & $1.00 \pm 0.24$ & & $0.98 \pm 0.28$ & & & \\
\hline Post-test & $1.07 \pm 0.16$ & & $0.71 \pm 0.18$ & & & \\
\hline $95 \% \mathrm{Cl}$ & $1.09,0.95$ & & $0.78,0.64$ & & & \\
\hline Dynamic balance (general index) & & $-1.00(0.33)$ & & $9.47(0.001)^{*}$ & $25.73(0.001)^{*}$ & 0.6 \\
\hline Pre-test & $70.98 \pm 2.14$ & & $67.33 \pm 1.25$ & & & \\
\hline Post-test & $69.92 \pm 2.14$ & & $73.74 \pm 2.63$ & & & \\
\hline $95 \% \mathrm{Cl}$ & $2.89,2.69$ & & $10.53 \pm 1.12$ & & & \\
\hline Lateral Jump, s & & $0.77(0.64)$ & & $8.36(0.001)$ & $12.67(0.02)$ & 0.70 \\
\hline Pre-test & $10.82 \pm 1.05$ & & $10.53 \pm 1.12$ & & & \\
\hline Post-test & $11.04 \pm 0.75$ & & $7.72 \pm 0.70$ & & & \\
\hline $95 \% \mathrm{Cl}$ & $10.41 \pm 1.65$ & & $8.52 \pm 2.4$ & & & \\
\hline Triple hop test, m & & $1.13(0.91)$ & & & & \\
\hline Pre-test & $3.91 \pm 0.29$ & & $4.24 \pm 0.23$ & & & \\
\hline Post-test & $3.92 \pm 0.31$ & & $4.82 \pm 0.25$ & & & \\
\hline $95 \% \mathrm{Cl}$ & $3.12 \pm 0.21$ & & $4.89 \pm 0.38$ & & & \\
\hline
\end{tabular}

ments, rehabilitation exercises were more effective, but the improvement was significant in both training groups, compared with the control group [22]. Babadi et al. also investigated the effect of a virtual exercise program on motor coordination in the elderly. These authors concluded that virtual exercises improved motor coordination in the elderly, and virtual reality-based training could be used as a new and attractive training method to improve the elderly's coordination in the daily programs of nursing homes [23].

The effectiveness of virtual reality-based training that was confirmed in the present study, can be attributed to several factors. Balance is a multifactorial issue influenced by various factors, such as strength, the depth of feeling, the speed of reaction, and movement [35].

Previous studies have shown that the exercises of a hybrid nature more significantly affects balance, compared with the exercises that emphasize only one variable $[35,36]$. The multifactorial nature of equilibrium can well confirm this statement. It seems logical that virtual reality-based training can improve balance by influenc- ing several important and influential factors on balance, namely power, proprioception, reaction speed, and the coordination of movement at the same time [19].

In a review study of balance and neuromuscular training, Habcher et al. concluded that the use of balance and neuromuscular training improved neuromuscular communication. It also significantly reduced acute wrist and knee injuries by reducing latency function. Therefore, performing such exercises is effective due to better neuromuscular coordination and the increased coordination of visual, vestibular, and sensory systems. It is an excellent exercise to improve the balance of athletes. Thus, the exercises designed in this study might improve balance by increasing the efficiency of sensory receptors and facilitating neuromuscular receptors, while improving joint proprioception [37].

To prevent injury, it is suitable to design an exercise program to strengthen balance, improve postural oscillation, and increase proprioception accuracy. Besides, this exercise program is safer and faster for the athlete to return to the sports scene. In the present study, the 
athletes were selected from the fields of basketball, volleyball, football, and handball. These disciplines include the most non-collision injuries of the lower extremities, such as zigzag movements, shear generative maneuvers, and jumping and landing mechanisms. Therefore, to improve performance balance, a research training protocol requires jumping and landing on one and two legs, maneuvers, and zigzag and shear movements without the loss of balance and agility in the frontal and sagittal directions [38].

Functional movements, such as jumping, landing, rotating, and shearing are defined as part of the specific functional repertoire of each individual. Functional capacity is the part of the brain's motor repertoire, in which specific movements are referred to as general skills (walking, sitting, running, etc), and others are classified as special skills (specialized movements in various sports). Functional rehabilitation helps people regain functional capacity by the motor repertoire of their brain [39]. Regarding sports injury rehabilitation, Lederman suggests that in the injury rehabilitation phase, it is better to use those exercises that exist in the person's movement repertoire and the person is relatively familiar with them [40]. Also, he states that neuromuscular training used in the rehabilitation phase of injury must have five characteristics:

First, the chosen exercises must be known to the person, and the person performs the movements with the full knowledge of the purpose of the exercise. According to the training protocol, the present study tried to use movements that are common in handball, basketball, and volleyball, and the person has full experience and knowledge in performing the movements. In this regard, we can refer to various jumping and shear movements used in the present study.

Second, the exercises should be performed actively, and the person should be involved in performing the movements.

The third characteristic expressed by Lederman is the provision of feedback to the athlete. Receiving verbal, observational, or internal feedback (proprioception) during exercise time will make the exercises more effective, also, the athlete will perform the movements correctly. The present study should be used as an observation (paying attention to the character performing the movement).

The fourth feature is that the subjects have to do the exercises with the necessary repetition to achieve the desired effectiveness.
The last feature is the principle of the similarity of training, which is one of the most crucial neuromuscular training requirements. It means that the exercises should be similar to what a person does on the field or when calling for movement needs [40]. The exercises designed in the present study largely provide the ability to simulate the movements used in the sports of handball, basketball, and volleyball.

In the protocol used in the present study, attempts have been made to design movements that can be performed at all three levels and the axes of movement. Also, in all designed movements, observing the characteristics of Lederman rehabilitation exercises reported above was considered the most effective exercise. The functional tests used in the present study are such that they require muscle involvement in all motor axes. Therefore, the positive effect of virtual reality-based training can be attributed to the factors affecting the quality of neuromuscular training. Finally, it is essential to note that there is no conclusive evidence that new virtual reality-based training affects athletes with functional ankle instability. In this regard, more studies are needed.

\section{Conclusion}

Virtual reality-based training can improve static and dynamic balance and performance in athletes with functional ankle sprains. Based on the present study results, trainers and specialists can use these exercises in medical and physiotherapy centers. One of the limitations of this study was commuting studying subjects to a suitable place to perform exercises and a small number of subjects. Also, future research could use implications for virtual reality-based training in different age groups and on different subjects and athletes from different disciplines to prevent injury.

\section{Ethical Considerations}

\section{Compliance with ethical guidelines}

This research project has been carried out at Kharazmi University conducted in February 2017. All ethical principles have been observed.

\section{Funding}

This research did not receive any grant from funding agencies in the public, commercial, or non-profit sectors. 


\section{Authors' contributions}

All authors contributed equally in preparing all parts of the research.

\section{Conflict of interest}

The authors declared no conflict of interest.

\section{References}

[1] Kalantariyan M, Minoonejad H, Rajabi R, Beyranvand R, Zahiri A. [The comparison of the electromyography activity of selected muscles of the ankle joint in athletes with ankle dorsiflexion range of motion limitation with healthy athletes during the single-leg jump landing (Persian)]. The Scientific Journal of Rehabilitation Medicine. 2013; 2(2):14-23. http:/ / medrehab.sbmu.ac.ir/article_1100141_en.html

[2] Kobayashi T, Gamada K. Lateral ankle sprain and chronic ankle instability: A critical review. Foot \& Ankle Specialist. 2014; 7(4):298-326. [DOI:10.1177/1938640014539813] [PMID]

[3] Wikstrom EA, Hubbard-Turner T, McKeon PO. Understanding and treating lateral ankle sprains and their consequences. Sports Medicine. 2013; 43(6):385-93. [DOI:10.1007/ s40279-013-0043-z] [PMID]

[4] Freeman M, Dean M, Hanham I. The etiology and prevention of functional instability of the foot. The Journal of Bone and Joint Surgery. 1965; 47(4):678-85. [DOI:10.1302/0301620X.47B4.678]

[5] Tropp H, Odenrick P, Gillquist J. Stabilometry recordings in functional and mechanical instability of the ankle joint. International Journal of Sports Medicine. 1985; 6(03):180-2. [DOI:10.1055/s-2008-1025836] [PMID]

[6] Delahunt E, Monaghan K, Caulfield B. Changes in lower limb kinematics, kinetics, and muscle activity in subjects with functional instability of the ankle joint during a single leg drop jump. Journal of Orthopaedic Research. 2006; 24(10):1991-2000. [DOI:10.1002/jor.20235] [PMID]

[7] Abbasi H, Alizadeh MH, Daneshmandi H, Barati AH. Comparing the effect of functional, extra-functional and combined exercises on dynamic balance in athletes with functional ankle instability. Sport Medicine Studies. 2015; 7(17):15-34. https://smj.ssrc.ac.ir/article_570_en.html

[8] Borreani S, Calatayud J, Martin J, Colado CJ, Tella V, Behm D. Exercise intensity progression for exercises performed on unstable and stable platforms based on ankle muscle activation. Gait \& Postur. 2014; 39(1):404-9. [DOI:10.1016/j.gaitpost.2013.08.006] [PMID]

[9] Gribble PA, Hertel J, Plisky P. Using the Star Excursion Balance Test to assess dynamic postural-control deficits and outcomes in lower extremity injury: A literature and systematic review. Journal of Athletic Training. 2012; 47(3):339-57. [DOI:10.4085/1062-6050-47.3.08] [PMID] [PMCID]
[10] Hale SA, Hertel J, Olmsted-Kramer LC. The effect of a 4-week comprehensive rehabilitation program on postural control and lower extremity function in individuals with chronic ankle instability. Journal of Orthopaedic \& Sports Physical Therapy. 2007; 37(6):303-11. [DOI:10.2519/ jospt.2007.2322] [PMID]

[11] Karimizadeh Ardakani M, Alizade MH, Ebrahimi Takamjani E. [The effect of 6-week hopping exercises program on joint position sense in athletes with functional ankle instability (Persian)]. Journal of Research in Rehabilitation Research. 2013; 9(3):540-52. http://jrrs.mui.ac.ir/index.php/jrrs/article/view/965

[12] Samadi H, Rajabi R, Karimizadeh Ardakani M. [The effect of six weeks of neuromuscular training on joint position sense and lower extremity function in male athletes with functional ankle instability (Persian)]. Journal of Exercise Science and Medicine. 2017; 9(1):15-33. [DOI:10.22059/JSMED.2017.62868]

[13] National Research Council (US) Steering Committee for the Workshop on Technology for Adaptive Aging, Pew RW, van Hemel SB. Technology for adaptive aging. Washington (DC): National Academies Press; 2004. [DOI:10.17226/10857] [PMID]

[14] Helal A, Mokhtari M, Abdulrazak B. The engineering handbook of smart technology for aging, disability and independence. Hoboken: John Wiley \& Sons; 2008. https:// books.google.com/books?id=NpldsRcAY9oC\&dq

[15] Adamovich SV, Merians AS, Boian R, Lewis JA, Tremaine $M$, Burdea GS, et al. A virtual reality-based exercise system for hand rehabilitation post-stroke. Presence: Teleoperators \& Virtual Environments. 2004; 14(2):161-74. [DOI:10.1162/1054746053966996]

[16] Anderson GS, Gaetz M, Holzmann M, Twist P. Comparison of EMG activity during stable and unstable push-up protocols. European Journal of Sport Science. 2013; 13(1):42-8. [DO I:10.1080/17461391.2011.577240]

[17] Bisson E, Contant B, Sveistrup H, Lajoie Y. Functional balance and dual-task reaction times in older adults are improved by virtual reality and biofeedback training. Cyberpsychology \& Behavior. 2007; 10(1):16-23. [DOI:10.1089/ cpb.2006.9997] [PMID]

[18] Cho GH, Hwangbo G, Shin HS. The effects of virtual realitybased balance training on balance of the elderly. Journal of Physical Therapy Science. 2014; 26(4):615-7. [DOI:10.1589/ jpts.26.615] [PMID] [PMCID]

[19] Goble DJ, Cone BL, Fling BW. Using the Wii Fit as a tool for balance assessment and neurorehabilitation: The first half decade of "Wii-search". Journal of Neuroengineering and Rehabilitation. 2014; 11(1):12. [DOI:10.1186/1743-0003-1112] [PMID] [PMCID]

[20] Merians AS, Jack D, Boian R, Tremaine M, Burdea GC, Adamovich SV, et al. Virtual reality-augmented rehabilitation for patients following stroke. Physical Therapy. 2002; 82(9):898-915. [DOI:10.1093/ptj/82.9.898] [PMID]

[21] Kim KJ, Heo M. Effects of virtual reality programs on balance in functional ankle instability. Journal of Physical Therapy Science. 2015; 27(10):3097-101. [DOI:10.1589/ jpts.27.3097] [PMID] [PMCID] 
[22] Kim K, Choi B, Lim W. The efficacy of virtual reality assisted versus traditional rehabilitation intervention on individuals with functional ankle instability: A pilot randomized controlled trial. Disability and Rehabilitation: Assistive Technology. 2019; 14(3):276-80. [DOI:10.1080/17483107.2018.1429501] [PMID]

[23] Yousefi Babadi S, Daneshmandi H. [Effect of a virtual reality training program (exergaming) on the motor coordination of the elderly (Persian)]. Scientific Journal of Rehabilitation Medicine. 2019; 8(2):169-76. [DOI:10.22037/JRM.2018.111268.1877]

[24] Zarei, M, Alizadeh MH, Rahnama N, Seif-Barghi T. [The Effect of FIFA warm-up comprehensive program 11+ on performance and physical fitness of Iranian adolescent male soccer players (Persian)]. Journal of Exercise Science and Medicine. 2016; 8(1):15-36. [DOI:10.22059/JSMED.2016.58861]

[25] Delahunt E, Coughlan GF, Caulfield B, Nightingale EJ, Christine Lin CW, Hiller CE. Inclusion criteria when investigating insufficiencies in chronic ankle instability. Medicine \& Science in Sports \& Exercise. 2010; 42(11):2106-21. [DOI:10.1249/ MSS.0b013e3181de7a8a] [PMID]

[26] Gribble PA, Delahunt E, Bleakley C, Caulfield B, Docherty CL, Fourchet $\mathrm{F}$, et al. Selection criteria for patients with chronic ankle instability in controlled research: A position statement of the International Ankle Consortium. The Journal of Orthopaedic and Sports Physical Therapy. 2013; 43(8):585-91. [DOI:10.2519/ jospt.2013.0303] [PMID]

[27] Huang PY, Lin CF. Effects of balance training combined with plyometric exercise in postural control: Application in individuals with functional ankle instability. Paper presented at: 6th World Congress of Biomechanics (WCB 2010). 1-6 August 2010; Singapore. https://link.springer.com/chapter/10.1007/978-3-642-14515-5_60

[28] Myer GD, Ford KR, McLean SG, Hewett TE. The effects of plyometric versus dynamic stabilization and balance training on lower extremity biomechanics. The American Journal of Sports Medicine. 2006; 34(3):445-55. [DOI:10.1177/0363546505281241] [PMID]

[29] Jorgensen M, Rathleff MS, Laessoe U, Caserotti P, Nielsen OBF, Aagaard P. Time-of-day influences postural balance in older adults. Gait \& Posture. 2012; 35(4):653-7. [DOI:10.1016/j.gaitpost.2011.12.018] [PMID]

[30] Pollock K, Gribble P, Pietrosimone B. The star excursion balance test as a predictor of lower extremity injury in high school football players [Internet]. 2010 [Updated 2010]. Available from: https://www.semanticscholar.org/paper/A-Thesisentitled-The-Star-Excursion-Balance-Test-a-Pollock-Gribble/34 2a49c0c06dbbc2eaa30be631721eba4c3fc5a8

[31] Ortiz A, Olson SL, Roddey TS, Morales J. Reliability of selected physical performance tests in young adult women. The Journal of Strength \& Conditioning Research. 2005; 19(1):39-44. [DOI:10.1519/00124278-200502000-00008] [PMID]

[32] Caffrey E, Docherty CL, Schrader J, Klossner J. The ability of 4 single-limb hopping tests to detect functional performance deficits in individuals with functional ankle instability. Journal of Orthopaedic \& Sports Physical Therapy. 2009; 39(11):799-806 [DOI:10.2519/jospt.2009.3042] [PMID]

[33] Hamilton RT, Shultz SJ, Schmitz RJ, Perrin DH. Triplehop distance as a valid predictor of lower limb strength and power. Journal of Athletic Training. 2008; 43(2):144-51. [DOI:10.4085/1062-6050-43.2.144] [PMID] [PMCID]
[34] Feng J, Chen K, Zhang C, Li H. A virtual reality-based training system for ankle rehabilitation. Paper presented at: 2018 IEEE International Conference on Progress in Informatics and Computing (PIC). 14-16 December 2018; China, Suzhou. [DOI:10.1109/PIC.2018.8706143]

[35] Farsi A, Abdoli B, Baraz P. [Effect of balance, strength, and combined training on the balance of the elderly women (Persian)]. Salmand: Iranian Journal of Ageing. 2015; 10(3):54-61. http:/ / salmandj.uswr.ac.ir/article-1-726-en.html

[36] Nagy E, Feher-Kiss A, Barnai M, Domján-Preszner A, Angyan L, Horvath G. Postural control in elderly subjects participating in balance training. European Journal of Applied Physiology. 2007; 100(1):97-104. [DOI:10.1007/s00421-0070407-x] [PMID]

[37] Hübscher M, Zech A, Pfeifer K, Hänsel F, Vogt L, Banzer W. Neuromuscular training for sports injury prevention: A systematic review. Medicine and Science in Sports and Exercise. 2010; 42(3):413-21. [DOI:10.1249/MSS.0b013e3181b88d37] [PMID]

[38] Holm I, Tveter AT, Fredriksen PM, Vøllestad M. A normative sample of gait and hopping on one leg parameters in children 7-12 years of age. Gait \& Posture. 2009; 29(2):317-21. [DOI:10.1016/j.gaitpost.2008.09.016] [PMID]

[39] Aslani M, Minoonejad H, Rajabi R. Comparing the effect of trx exercise and hoping on balance in male university student athletes. Physical Treatments: Specific Physical Therapy Journal. 2018; 7(4):241-50. [DOI:10.32598/ptj.7.4.241]

[40] Lederman E. Neuromuscular rehabilitation in manual and physical therapy: Principles to practice. London: Churchill Livingstone; 2010. [DOI:10.1016/B978-0-443-06969-7.00014-0] 